\title{
The influence of an epigenetics diet on the cancer epigenome
}

\author{
Kayla A Lewis ${ }^{1} \&$ Trygve O Tollefsbol ${ }^{*}, 1,2,3,4,5$ \\ ${ }^{1}$ Department of Biology, University of Alabama at Birmingham, Birmingham, AL 35294, USA \\ ${ }^{2}$ Comprehensive Cancer Center, University of Alabama at Birmingham, Birmingham, AL 35294, USA \\ ${ }^{3}$ Comprehensive Center for Healthy Aging, University of Alabama at Birmingham, Birmingham, AL 35294, USA \\ ${ }^{4}$ Nutrition Obesity Research Center, University of Alabama at Birmingham, Birmingham, AL 35294, USA \\ ${ }^{5}$ Comprehensive Diabetes Center, University of Alabama at Birmingham, Birmingham, AL 35294, USA \\ * Author for correspondence: Tel.: +1 205934 4573; Fax: +1 205975 6097; trygve@uab.edu
}
'There have been many advances made on the interactions between dietary compounds and the epigenome and here we explore some of the exciting and emerging topics on the epigenetics diet."

First draft submitted: 19 June 2017; Accepted for publication: 26 June 2017; Published online: 11 August 2017

Keywords: $5 \mathrm{hmC} \cdot$ acetylation - cancer $\bullet$ DNA methylation - epigenetic diet $\bullet$ histone modifications •
microbiome $\bullet$ phytochemicals

We coined the term 'epigenetics diet' only 6-years ago based on numerous studies delineating the impact of bioactive dietary compounds on changes in the epigenome [1]. There have been many advances made on the interactions between dietary compounds and the epigenome and here we explore some of the exciting and emerging topics on the epigenetics diet. There is an aberrant gene expression due to epigenetic changes in all cancer types, so an intuitive and holistic approach to cancer therapy would be to use these bioactive dietary compounds as a means of not only neutralizing epigenomic aberrations as cancer treatment, but also as cancer prevention. Aberrant gene expression can be due to changes in DNA methylation and histone acetylation, among others.

\section{Combinatorial epigenetic-modifying phytochemicals}

There are limitations when considering use of dietary components to treat or prevent cancer. Although, many of these phytochemicals are efficacious alone or in combination, some are only efficacious at considerably high doses not achievable by diet alone, unless it is the only food consumed. In a more realistic view of dietary phytochemicals, an increasing number of current studies focus on their combination. Humans generally do not simply eat one food item, so combinatorial studies are important to enhance our understanding of the interactions between various epigenomic-modifying dietary compounds. There will be a future in this combinatorial approach of the epigenetics diet simply because of the sheer quantity of combinations possible. Compounds that display anticancer properties by themselves may act in an additive, synergistic or even in antagonistic manner in a combination $[2,3]$. The epigenetic mechanisms for these interactions are not fully understood and are an area of increasing interest for our laboratory as well as many others.

\section{Epigenetics diet \& the gut microbiome}

Another topic that has been increasing in popularity with respect to the epigenetics diet is the microbiome. Low molecular weight byproducts generated from the gut flora have the ability to influence the epigenome through DNA modifications as well as chromatin remodeling [4]. Bacterial metabolites, like folate, influence DNA methylation through their ability to donate methyl moieties to $S$-adenosylmethionine (SAMe), which in turn provides the methyl groups for DNA methyltransferases to generate 5 -methylcytosine $(5 \mathrm{mC})$. Members of the gut flora can produce the choline metabolite trimethylamine $\mathrm{N}$-oxide (TMAO), which can also donate methyl groups to SAMe. This metabolite has also been shown to contribute to cardiac dysfunction in Western diet-induced obese mice [15]. Butyrate and other B vitamins are commonly produced by the gut microbiome. Butyrate is an epigenetic modifier 
primarily known for its role in histone deacetylase (HDAC) inhibition, but it also has a lesser-known role in DNA methylation. Interestingly, metabolic diseases can be transferred using fecal transplants, so it is possible to assume that the gut microbiome is a large source of important coenzymes and vitamins. The intestines absorb the generated water-soluble vitamins. This could have significant implications when considering epigenetic changes in colorectal cancers, for instance [14]. Arumugan et al. characterized the composition and function of gut microbiome members and actually determined that there was an enterotype cluster that not only had elevated levels of biotin synthesis enzymes, but also enzymes necessary for vitamin $C$ synthesis, a vitamin that acts as a co-factor in the DNA demethylation process [10].

\section{Hydroxymethylation of DNA: no longer considered solely an intermediate of DNA demethylation}

Both the microbiome and combinatorial epigenetic-modifying phytochemicals influence the prevalence of 5hydroxymethylcytosine $(5 \mathrm{hmC})$. The $5 \mathrm{hmC}$ in DNA demethylation is an oxidized intermediate in the conversion of $5 \mathrm{mC}$ to an unmethylated state by the ten-eleven translocation 1 enzyme (TET1). $5 \mathrm{hmC}$ may be an epigenetic modification in its own right, maintaining the ability to recruit chromatin or transcriptional modifiers to the site. $5 \mathrm{hmC}$ and TET1 undergo changes in embryonic stem cells and with cellular differentiation, and the loss of $5 \mathrm{hmC}$ is considered a hallmark of cancer [5,6]. Once differentiation is completed, the levels of the TET enzymes, particularly TET1 and TET2, decrease [13]. It is notable that vitamin $\mathrm{C}$ is a potential co-factor for Fe(II) 2-oxoglutarate dioxygenase enzymes like TET and can, therefore, enhance demethylation of genes through the activation of TET [7]. Common dietary sources of vitamin $\mathrm{C}$ include oranges, red and green peppers, kale, broccoli and guava. As aforementioned, vitamin $\mathrm{C}$ and butyrate can be produced by the gut microbiome. Chronic administration of sodium butyrate to the genetic rat model for depression resulted in an increase in the TET1 enzyme and also an increase in $5 \mathrm{hmC}$ [11]. The common tissue studied after treatment with a TET enzyme activator has been brain tissue, although these results show promise for various cancer treatments and/or prevention as well.

Considering diabetes and diet, transgenerational studies have shown that lean offspring of non-high fat diet-fed mothers resulted in a reduction of $5 \mathrm{mC}$ and an increase of $5 \mathrm{hmC}$ within the promoter of Pomc (proopiomelanocortin) [8]. POMC is a hypothalamic anorexigenic neuropeptide involved in feeding regulation. The process of DNA demethylation is a signal transduction pathway $(5 \mathrm{mC}$ is converted to $5 \mathrm{hmC}$, which is converted to 5-formylcytosine and 5-carboxylcytosine that converts to unmodified cytosine), with the final product resulting in gene transcription. Therefore, an increase in the enzymes (TET1, TET2 and TET3) due to an increase in the necessary co-factor will result in more product formation. Because vitamin C acts as a co-factor of the TET family of enzymes, dietary vitamin C has the same result as a known DNMT inhibitor, such as 5-aza-2'-deoxycytidine. Other dietary DNMT inhibitors such as epigallocatechin gallate and genistein that are components of the epigenetics diet may in fact promote this intermediate, which is indistinguishable in a common bisulfite assay for DNA methylation content. TET hydroxylase activity assays are one way to measure enzyme activity, while TAB-seq (TET-assisted bisulfite sequencing) will distinguish between $5 \mathrm{hmC}$ and $5 \mathrm{mC}$. In an epigenome-wide study comparing regular kidney cells with clear cell renal cell carcinoma, it was actually determined that loss of $5 \mathrm{hmC}$ was linked to gene body hypermethylation upon TAB-seq analysis. Interestingly, there were no differences in the levels of $5 \mathrm{mC}$ [12].

It appears that some housekeeping genes do not utilize $5 \mathrm{hmC}$ for gene regulation (e.g., those encoding for mitochondrial and ribosomal proteins), while levels of $5 \mathrm{hmc}$ are highly tissue-specific; brain regions derived from the same embryonic structure have similar $5 \mathrm{hmc}$ levels [9]. Therefore, epigenetic-modifying dietary compounds that affect the TET family of enzymes will probably not have an effect on housekeeping genes, but will mainly activate genes that are expressed differentially between tissue types. We propose that much like DNA methylation and histone modifications, DNA hydroxymethylation is a form of epigenetic modification to the genome that is important for gene regulation. Genes that did not appear to be differentially methylated using bisulfite sequencing will need to be re-evaluated using TAB-seq considering the implications of active gene transcription and the presence of $5 \mathrm{hmC}$ within the promoter and enhancer regions. It will be important to re-evaluate previous studies that only used levels of $5 \mathrm{mC}$ as a marker of DNA methylation and to assess the role of the epigenetics diet for its impact on key genes that may be regulated by changes in $5 \mathrm{hmC}$ content.

\section{Digesting the scope of dietary epigenetics \& cancer}

The nutrient data laboratory generated by the USDA is an exhaustive and growing database accessible by the general public that characterizes the composition of foods. With this information, personalized diets can be self- 
manageable. However, interactions between epigenetic-modifying foods still need to be determined. Once more is known about the interactions between various phytochemicals, more informed epigenetic dietary regimens will be feasible.

The gut microbiome can also influence these interactions due to metabolite production. Fecal transplants have been used to successfully treat recurrent Clostridium difficile infections and may show promise for cancer patients. For example, preliminary studies from our laboratory show that genistein impacts the composition of the microbiome in mice that have been humanized with fecal transplants from breast cancer patients.

Very little is known regarding the transcriptional regulation of the TET family of proteins, so there is mounting interest in determining the effects of epigenetic-modifying dietary phytochemicals and the TET family promoter regions. It will also be important to determine the direct effects of these phytochemicals and hydroxymethylation status.

These exciting and emerging areas of the epigenetics diet, as well as others, are likely to reveal important translational information for cancer prevention and therapy. The future is indeed bright for the role of the epigenetics diet and its impact on the cancer epigenome.

\section{Financial \& competing interests disclosure}

This work was supported in part by the National Institutes of Health (NCI: RO1 CA17844 and RO1 CA2044346) and the American Institute for Cancer Research to TOT, as well as the Susan G. Komen Graduate Training in Disparities Research grant to KA Lewis. The authors have no other relevant affiliations or financial involvement with any organization or entity with a financial interest in or financial conflict with the subject matter or materials discussed in the manuscript apart from those disclosed.

No writing assistance was utilized in the production of this manuscript.

\section{References}

1 Hardy TM, Tollefsbol TO. Epigenetic diet: impact on the epigenome and cancer. Epigenomics 3(4), 503-518 (2011).

2 Efferth T, Koch E. Complex interactions between phytochemicals. The multi-target therapeutic concept of phytotherapy. Curr. Drug Targets 12(1), 122-132 (2011).

3 Phan MA, Paterson J, Bucknall M, Arcot J. Interactions between phytochemicals from fruits and vegetables: effects on bioactivities and bioavailability. Crit. Rev. Food Sci. Nutr. doi:10.1080/10408398.2016.1254595 (2016) (Epub ahead of print).

4 Paul B, Barnes S, Demark-Wahnefried W et al. Influences of diet and the gut microbiome on epigenetic modulation in cancer and other diseases. Clin. Epigenetics 7, 112 (2015).

5 Ito S, D’Alessio AC, Taranova OV, Hong K, Sowers LC, Zhang Y. Role of TET proteins in $5 \mathrm{mC}$ to 5 hmC conversion, ES-cell self-renewal and inner cell mass specification. Nature 466(7310), 1129-1133 (2010).

$6 \mathrm{Hu} \mathrm{H}$, Shu M, He L, Yu X et al. Epigenomic landscape of 5-hydroxymethylcytosine reveals its transcriptional regulation of lncRNAs in colorectal cancer. Brit. J. Canc. 116, 658-668 (2017).

7 Minor EA, Court BL, Young JI, Wang G. Ascorbate induces ten-eleven translocation (TET) methylcytosine dioxygenase-mediated generation of 5-hydroxymethylcytosine. J. Biol. Chem. 288(19), 13669-13674 (2013).

8 Marco A, Kisliouk T, Tabachnik T, Weller A, Meiri N. DNA CpG methylation $(5 \mathrm{mc})$ and its derivative ( 5 hmc) alter histone post translational modifications at the Pomc promoter, affecting the impact of perinatal diet on leanness and obesity of the offspring. Diabetes 65(8), 2258-2267 (2016).

9 Lin I-H, Chen Y-F, Hsu M-T. Correlated 5-hydroxymethylcytosine (5hmC) and gene expression profiles underpin gene and organ-specific epigenetic regulation in adult mouse brain and liver. PLoS ONE 12(1), doi:10.1371/journal.pone.0170779 (2017) (Epub ahead of print).

10 Arumugam M, Raes J, Pelletier E et al. Enterotypes of the human gut microbiome. Nature 473(7346), 174-180 (2011).

11 Wei YB, Melas PA, Wegener G, Mathé AA, Lavebratt C. Antidepressant-like effect of sodium butyrate is associate with an increase in TET1 and 5-hydroxymethylation levels in the Bdnf gene. Int. J. Neuropsychopharmacol. 18(2), pii:pyu032 (2015).

12 Chen K, Zhang J, Guo Z et al. Loss of 5-hydroxymethylcytosine is linked to gene body hypermethylation in kidney cancer. Cell Res. 26(1), 103-118 (2016).

13 Koh KP, Yabuuchi A, Rao S et al. TET1 and TET2 regulate 5-hydroxymethylcytosine production and cell lineage specification in mouse embryonic stem cells. Cell Stem Cell 8(2), 200-213 (2011).

14 Vogtmann E, Hua X, Zeller G et al. Colorectal cancer and the human gut microbiome: reproducibility with whole-genome shotgun sequencing. PLoS ONE 11(5), e0155362 (2016).

15 Chen K, Zheng X, Feng M, Li D, Zhang H. Gut microbiota-dependent metabolite trimethylamine N-oxide contributes to cardiac dysfunction in western diet-induced obese mice. Front. Physiol. 8, 139 (2017). 
